\title{
Frequency of 6-week follow-up appointment scheduling after intrauterine device insertion
}

\author{
Abigail Bernard, Catherine L Satterwhite, Madhuri Reddy
}

School of Medicine, University of Kansas, Kansas City, Missouri, USA

\section{Correspondence to} Abigail Bernard, School of Medicine, University of Kansas, Kansas City, MO 66160, USA; abernard3@kumc.edu

Received 3 April 2017 Revised 6 October 2017 Accepted 10 October 2017 Published Online First 16 November 2017

\begin{abstract}
Introduction Long-acting reversible contraception (LARC) is widely recommended to reduce unintended pregnancy in the USA. As intrauterine device (IUD) use increases, evaluating the role of post-insertion follow-up is important. Methods A retrospective patient record review was conducted to assess the follow-up experience of women who had an IUD placed at the University of Kansas Medical Center from 1 January to 30 June 2015. Data were collected on patient demographics, IUD placement, follow-up visit attendance, and outcomes in the 12 months following placement. The primary outcome of interest was the proportion of patients who attended a 6-week follow-up visit. Secondary outcomes included adverse events detected at the 6-week visit and IUD removal within a year of placement.

Results Among 380 women who had an IUD inserted, physician documentation of a recommended 6-week follow-up visit was present in $91.3 \%$ of patient medical records. Two-thirds $(66.6 \%)$ of patients receiving a recommendation returned for a follow-up visit. Of the 380 women who had an IUD placed, 66 (17.4\%) had their IUD removed within 1 year of placement. Of those, 50 women attended the 6-week follow-up visit and 16 did not (19.8\% vs $12.6 \%, p=0.08)$. Of the IUD removals, 14 occurred at the 6 -week visit. After excluding IUD removals which occurred at the 6-week visit, attending a 6-week follow-up visit was not associated with IUD removal or retention $(p=0.52)$.
\end{abstract}

Conclusion Despite recommendations to forgo the 6-week follow-up visit, visits were still common, with no demonstrated value added.

\section{INTRODUCTION}

Almost half of pregnancies in the USA are unintended, with $82 \%$ of teenage pregnancies being unintended. ${ }^{1}$ While long-acting reversible contraception (LARC) options exist, these highly effective methods are not widely used globally. ${ }^{2}$ To promote their

\section{Key messages}

- Results from this study support the Centers for Disease Control and Prevention's recommendation to not routinely schedule a 6-week follow-up visit after intrauterine device (IUD) insertion.

- As providers continue to promote longacting reversible contraception methods as first-line contraception and IUD use increases, forgoing the 6-week follow-up visit should be a consideration.

use, the American College of Obstetricians and Gynecologists (ACOG) released 2012 guidelines recommending that LARCs, such as intrauterine devices (IUDs) and implants, are offered as the primary birth control option for sexually active adolescents. ${ }^{3}$ With these recommendations, ACOG broadly encouraged LARC use among all women of reproductive age.

Currently, LARC methods are recognised as the most effective form of birth control for sexually active women of reproductive age. ${ }^{3}$ IUDs are generally well tolerated, there are few side effects, and continuation of IUDs and implants is higher than continuation with other options, such as oral contraceptive pills, patches, rings or barrier methods. Studies have also shown that patient satisfaction with LARC use is high. ${ }^{4}$ Over the past 10 years, LARC use has increased approximately fivefold among women aged 15-44 years in the USA. In 2002, an estimated $1.5 \%$ of women aged 15-44 years utilised LARC methods; recent data suggest that $7.2 \%$ of women in 20112013 utilised a LARC method. ${ }^{5}$ Comparatively, among women aged $15-49$ years living in the UK, an estimated $11 \%$ utilised a LARC method in $2015 .^{6}$

To obtain a LARC, a patient must access a healthcare setting and have the device 
placed. While there is no manufacturer recommendation for a follow-up visit after implant placement, once an IUD has been placed, a 6-week follow-up visit is generally recommended by manufacturers. ${ }^{7-9}$ The U.S. Centers for Disease Control and Prevention (CDC) suggest that a woman should generally return to her healthcare provider at any time to discuss concerns including side effects. However, a routine follow-up visit is not recommended by the CDC, due to research on the value of a short-term follow-up visit after IUD insertion being limited and of low quality. ${ }^{10} \mathrm{~A}$ study published in Contraception in 2012 came to the same conclusion as the CDC regarding previous research. ${ }^{11}$ Additionally, in 2015, the UK Faculty of Sexual \& Reproductive Healthcare (FSRH) suggested that more priority be placed on adequately counselling women on which side effects to expect and encouraging women to return to the clinic if they have concerns, rather than offering a routine follow-up visit, which the FSRH stated is typically poorly attended. ${ }^{12}$ It is evident that there is a disconnect between manufacturers' and healthcare agencies' recommendations. ${ }^{10} 12$

As LARC use, demand and placement continues to increase, evaluating the corresponding clinical practice and flow is important, particularly in relation to post-insertion follow-up. The purpose of this study was to determine how often patients attend a 6-week follow-up visit after LARC placement at the University of Kansas Medical Center (KUMC) despite CDC recommendations against this practice, and to evaluate the outcomes of these visits.

\section{METHODS}

A retrospective medical record review of the follow-up visits of women who had an IUD placed from 1 January to 30 June 2015 was conducted. Eligible women were identified using the current procedural terminology (CPT) of IUD insertion. Data were collected on patient demographics, follow-up visit attendance, and outcomes in the first year following placement. The primary outcome of interest was the proportion of patients who attended a 6-week follow-up visit. The 6-week follow-up visit was defined as any visit that occurred between insertion and 3 months following insertion. One secondary outcome of interest included IUD removal within a year of placement. Institutional Review Board (IRB) approval was obtained. This research did not receive any specific grant from funding agencies in the public, commercial, or not-forprofit sectors.

\section{RESULTS}

Between 1 January and 30 June 2015, 380 women had an IUD placed at KUMC. The women's mean age was 29.3 (SD 6.4) years. Most women chose a hormonal IUD (levonorgestrel $52 \mu \mathrm{g}$ intrauterine system (IUS) 88.4\%, levonorgestrel $13.5 \mu \mathrm{g}$ IUS 2.1\%) (table 1). The vast majority $(91.3 \%)$ of patient medical records
Table 1 Characteristics of women receiving an intrauterine device and follow-up visit recommendation and attendance

\begin{tabular}{|c|c|c|c|}
\hline Characteristic & $\begin{array}{l}\text { Total }(\%) \\
(\mathrm{n}=380)\end{array}$ & $\begin{array}{l}\text { Follow-up visit } \\
\text { recommended } \\
(\%)(n=347)\end{array}$ & $\begin{array}{l}\text { Follow- } \\
\text { up visit } \\
\text { attended } \\
(\%)(n=253)\end{array}$ \\
\hline \multicolumn{4}{|l|}{ Age (years) } \\
\hline$<30$ & 55.0 & 55.0 & 55.3 \\
\hline$\geq 30$ & 45.0 & 45.0 & 44.7 \\
\hline \multicolumn{4}{|l|}{ Race } \\
\hline White & 62.3 & 61.3 & 64.3 \\
\hline Black & 18.7 & 18.8 & 16.3 \\
\hline Other & 19.0 & 19.9 & 19.4 \\
\hline Married & 41.3 & 41.5 & 38.9 \\
\hline \multicolumn{4}{|l|}{ Parity* } \\
\hline 0 & 35.2 & 35.9 & 39.4 \\
\hline$\geq 1$ & 64.8 & 64.1 & 60.6 \\
\hline First IUD $(\%)^{*}$ & 77.9 & 79.0 & 82.2 \\
\hline \multicolumn{4}{|l|}{ Type of IUD (\%) } \\
\hline $\begin{array}{l}\text { LNG IUS } 52 \mu \mathrm{g} / \\
\text { LNG IUS } 13.5 \mu \mathrm{g} \\
\text { (Mirena/Skyla) }\end{array}$ & 90.5 & 91.1 & 91.7 \\
\hline $\begin{array}{l}\text { Copper T 380A } \\
\text { (ParaGard) }\end{array}$ & 9.5 & 8.9 & 8.3 \\
\hline $\begin{array}{l}\text { No prior } \\
\text { contraception }\end{array}$ & 37.4 & 38.6 & 39.1 \\
\hline
\end{tabular}

contained physician documentation recommending a follow-up visit after IUD insertion (figure 1). Two-thirds $(66.6 \%)$ of the patients who received a recommendation returned for a 6-week follow-up visit.

At the follow-up visit, bleeding concerns were documented in $26.9 \%$ of visits. String concerns (i.e. the strings were too long, the strings were irritating to the partner) were documented in $9.1 \%$ of the visits. A total of 23 ultrasound scans were ordered before $(n=7)$ or at $(n=16)$ the 6 -week follow-up visit. The primary reason for ordering an ultrasound at the 6 -week visit was to confirm IUD placement $(n=16)$. Seven ultrasounds were ordered at other times as a result of patient complaints or Emergency Department presentation. Documentation of ultrasound completion existed in all patient medical records except one. None of the ultrasounds performed showed evidence of IUD expulsion. Two ultrasounds showed malpositioned devices, and one ultrasound showed a minute perforation.

Of the 380 women who had an IUD placed, 66 (17.4\%) had their IUD removed within 1 year of placement. Of the 344 levonorgestrel-releasing devices (LNG $52 \mu \mathrm{g}$ IUS and LNG $13.5 \mu \mathrm{g}$ IUS) placed, 61 (17.7\%) were removed. Of the 36 Copper T 380A 
Figure 1 Frequency of physician recommendations to attend 6-week follow-up visit and frequency of attendance. IUD, intrauterine device.

placed, $13.9 \%(5 / 36)$ were removed. The type of IUD was not associated with IUD removal $(p=0.56)$. Among women who attended a 6-week follow-up visit, $19.8 \%(\mathrm{n}=50)$ had their IUD removed within 1 year of placement, compared with $12.6 \%(n=16)$ of women who did not attend a 6 -week follow-up visit $(\mathrm{p}=0.08)$.

Of the 66 IUD removals, 14 (5.5\%) were removed at the 6 -week visit. Six of these were removed following patient request due to a variety of complaints (i.e. pelvic pain, desire for pregnancy); four were removed due to a malpositioned IUD (two IUDs were immediately replaced); three were expelled prior to the visit (one was replaced); and one removal was due to the presence of a septate uterus (physician recommendation against IUD). After excluding these 14 IUD removals, visit attendance was not associated with IUD removal; $15.1 \%$ of women who attended the 6-week visit had their IUD removed within 1 year (but outside of the 6-week visit, 36/239) compared with $12.6 \%$ of women who did not attend the 6-week visit (16/127) $(\mathrm{p}=0.52)$.

\section{DISCUSSION}

The results from this study support CDC's recommendation to not routinely schedule a 6-week follow-up visit after IUD insertion. Attending a 6-week follow-up visit is not associated with a reduced risk of IUD removal, and patient concerns raised at the visit were not common. The majority of the IUD removals occurred outside the 6-week follow-up window, suggesting that women made appointments due to specific IUD concerns, reflecting CDC's recommendation.

Most patient medical records contained no documentation of patients raising concerns discussed at the follow-up visit. The concerns that were documented related to bleeding duration and string length. Such concerns could potentially be managed via a telephone call instead of at the 6-week visit. Additionally, providers should deliver clear counselling at the time of insertion about what to expect in the months following insertion, paying particular attention to these concerns (i.e. bleeding concerns and string length), as suggested by FSRH's recommendations. ${ }^{12}$

Limitations of the study include reliance on the accuracy of medical record documentation to collect data, the small sample size (particularly for those using the copper IUD, making comparison of limited value), and the retrospective nature of the study. It is possible that some providers did not document specific patient concerns at their 6-week visit. Additionally, variation between clinic providers could have existed when determining which patient concerns, if any, to document. These results are also based on a single clinical setting and may not be generalisable to other settings. Areas for further research include obtaining the patient and physician perspectives regarding forgoing the visit. This study provided an objective look at the role of the 6-week follow-up visit, but the patient and physician perspectives were not obtained.

While review data suggest no clear benefit from attending a routine 6-week follow-up visit and CDC and FSRH recommendations do not encourage this practice, two-thirds of patients attended a visit. Considering the patient perspective, a 6-week follow-up appointment requires time, effort and an intrusive pelvic examination for an IUD check. These visits also consume staff time and other healthcare resources. As providers continue to promote LARC methods as first-line contraception and IUD use increases, forgoing the 6-week follow-up visit should be considered.

Contributors $\mathrm{AB}$ and CLS planned the study. MR provided clinical study oversight and facilitated access to electronic 
medical records. $\mathrm{AB}$ collected the data. $\mathrm{AB}$ and CLS performed data analysis. $\mathrm{AB}$ prepared the manuscript, and CLS and MR provided manuscript feedback. AB submitted the manuscript. CLS oversaw all study processes and outcomes.

Funding This research did not receive any specific grant from funding agencies in the public, commercial, or not-for-profit sectors.

Competing interests None declared.

Ethics approval Institutional Review Board (IRB)

Provenance and peer review Not commissioned; externally peer reviewed.

(C) Article author(s) (or their employer(s) unless otherwise stated in the text of the article) 2018. All rights reserved. No commercial use is permitted unless otherwise expressly granted.

\section{REFERENCES}

1 Baldwin MK, Edelman AB. The effect of long-acting reversible contraception on rapid repeat pregnancy in adolescents: a review. J Adolesc Health 2013;52:S47-53.

2 Ali MM, Sadler RK, Cleland J, et al. Long-term contraceptive protection, discontinuation and switching behaviour: intrauterine device (IUD) use dynamics in 14 developing countries. London: World Health Organization and Marie Stopes International, 2011.

3 IUDs and Contraceptive Implants Safe for Teens. Women's health care physicians. The American Congress of Obstetricians and Gynecologists, 2012.
4 Behringer T, Reeves MF, Rossiter B, et al. Duration of use of a levonorgestrel IUS amongst nulliparous and adolescent women. Contraception 2011;84:e5-10.

5 Branum AM, Jones J. Trends in long-acting reversible contraception use among U.S. women aged 15-44. NCHS Data Brief 2015;188:1-8.

6 Mohamed A, Sadler RK, Cleland J, et al. Long-term contraceptive protection, discontinuation and switching behavior: intrauterine device (IUD) use dynamics in 14 developing countries. London: World Health Organization and Marie Stopes International, 2011. www.who.int

7 Bayer. Questions and answers about Mirena. Mirena, 2017. https://www.mirena-us.com/q-and-a/ (accessed 10 Feb 2016).

8 Teva Women's Health, Inc. What to expect. PARAGARD (intrauterine copper contraceptive), 2017. http://paragard.com/ what-to-expect.aspx (accessed 10 Feb 2016).

9 Bayer. What to expect. Skyla, 2017. http://www.skyla-us.com/ getting-skyla/index.php (accessed 10 Feb 2016).

10 Curtis KM, Jatlaoui TC, Tepper NK, et al. U.S. Selected practice recommendations for contraceptive use, 2016. MMWR Recomm Rep 2016;65:1-66.

11 Steenland MW, Zapata LB, Brahmi D, et al. The effect of follow-up visits or contacts after contraceptive initiation on method continuation and correct use. Contraception 2013;87:625-30.

12 Faculty of Sexual and Reproductive Healthcare Clinical Guidance. Intrauterine Contraception: Clinical Effectiveness Unit: Royal College of Obstetricians and Gynaecologists, 2015. https://www.fsrh.org/standards-and-guidance/documents/ceu guidanceintrauterinecontraception/ (accessed 16 Jul 2017).

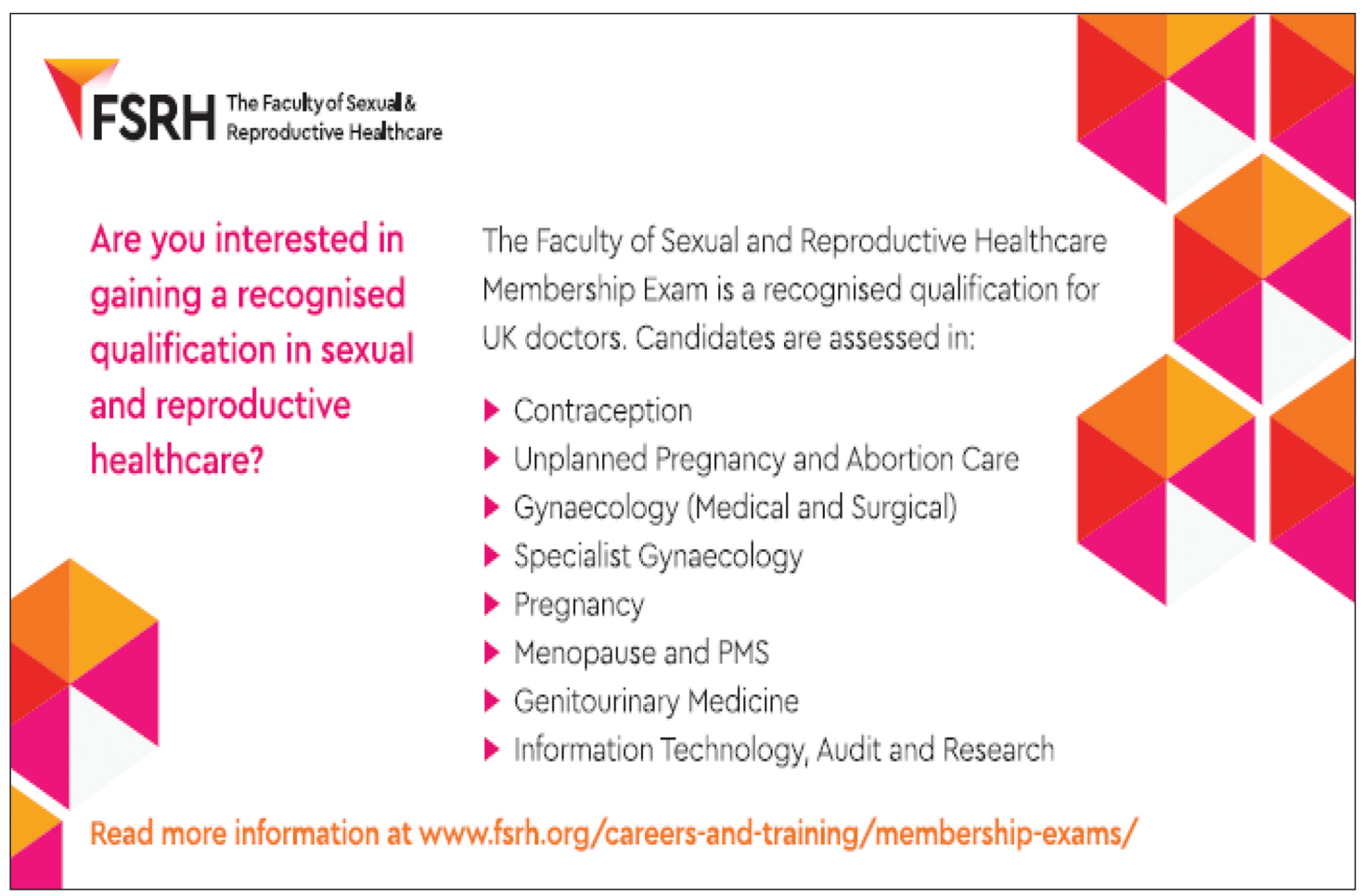

